\title{
Implementación de prácticas de laboratorio con costo mínimo
}

\section{(Implementation of laboratory practice with minimal cost)}

\author{
Idalberto Tamayo Ávila ${ }^{1}$, Luis Geovanny Pazmiño Bravo², Diego Fabián Valencia \\ Alvear $^{3}$, Mayra Mariuxi Galván Paredes ${ }^{4}$, Marcos Antonio Batista Zaldivar ${ }^{5}$
}

\begin{abstract}
Resumen:
En este trabajo se expone una iniciativa que consistió en implementar los montajes de 24 prácticas de laboratorio para las asignaturas de Física General, Física Aplicada, Calor y Electromagnetismo; en la Universidad Tecnológica Equinoccial (sede Santo Domingo). Para cumplir con el objetivo se realizaron tres tesis donde se concibieron las prácticas utilizando equipamiento almacenado que no se usaba; sumando a esto el aporte personal de los estudiantes se obtuvieron otros instrumentos para completar los montajes; cada práctica fue puesta a punto y validada para el uso docente mediante los métodos de cálculo de errores y regresión lineal. La culminación del trabajo significó un ahorro calculado en \$16.369,57 comparado con el hecho de si se hubiesen comprado cada uno de los montajes de las prácticas.
\end{abstract}

Palabras clave: laboratorio, habilidades experimentales, errores, validación, optimización.

\begin{abstract}
:
In this work is reported an initiative to implement 24 assembly laboratories for General Physics, Applied Physics and Heat and Electromagnetism, in the premises of the Universidad Tecnológica Equinoccial (Santo Domingo, Ecuador). For this purpose three student memoires where designed and performed with unused, stored equipment, plus some additional material coming from students contribution. Each practice was set up and validated for teaching purposes with the methods of calculation errors and linear regression. This work saved the institution $\$ 16,369.57$ compared with the cost of buying a commercial set for the laboratory.
\end{abstract}

Keywords: laboratory, experimental skills, errors, validation, optimization

\section{Introducción}

Las motivaciones para la realización de este trabajo estuvieron dadas en que la sede Santo Domingo nunca contó con un laboratorio de Física; por otra parte se percibió en los estudiantes de estas asignaturas la dificultad de interpretar conceptos físicos y aplicarlos; en otro orden de cosas, existía la necesidad de reformar los programas analíticos de ciencias básicas hasta el nivel que requiere la ingeniería, estando acorde con otras universidades nacionales e internacionales y

\footnotetext{
${ }_{1}^{1}$ Universidad Tecnológica Equinoccial, Quito - Ecuador (idalberto.tamayo@ute.edu.ec).

${ }^{2}$ CELEC EP TRANSELECTRIC, Ecuador (luis.pazmino@celec.gob.ec).

${ }^{3}$ Universidad Tecnológica Equinoccial, Santo Domingo - Ecuador (vadf502011@ute.edu.ec).

${ }^{4}$ CNEL SANTO DOMINGO, Ecuador (mayra.galvan@std.cnel.gob.ec).

${ }^{5}$ Unidad Educativa Virgilio Drouet, Ecuador (virgiliodrouet_comunicaciones@hotmail.com)
} 
de esta manera hacer una contribución para elevar la calidad de los programas desarrollando habilidades teóricas y experimentales (Agudelo, García, 2010).

Una práctica de laboratorio puede considerarse como una "pequeña investigación" donde los estudiantes aplican el método científico. La ingeniería es por naturaleza cuantitativa y no puede ser remplazada por palabras, por lo que deben adquirirse habilidades en el cálculo, medición, experimentación, interpretación de resultados, métodos y formas de expresarlos desde los primeros niveles de la carrera e incluso desde la enseñanza precedente. Por todo lo anterior la enseñanza teórica tiene que ir acompañada de la práctica y solo puede materializarse en un laboratorio real. (Flores, Caballero, Moreira, 2009).

Si los montajes para la realización de prácticas de laboratorio docentes se adquieren mediante la compra directa a un proveedor pueden resultar costosos por su exclusividad ya que no son fabricados en masa. Muchas veces se venden en kit y no se ajustan completamente a los intereses de un programa analítico de una asignatura específica.

Si las instituciones aprovechan las iniciativas de los docentes y estudiantes, estos montajes pueden concebirse e implementarse usando equipamiento almacenado en los laboratorios de institutos y universidades que conjuntamente con la compra aislada de otros componentes permite que la práctica montada se haga con un costo mínimo (Gil, 2015).

La experiencia que representa para un estudiante el hecho de poder concebir una práctica, montarla, experimentar, comparar si los resultados son válidos, mejorarla hasta ponerla a punto; contribuyen sin duda a su desarrollo profesional y puede ser el punto de partida para la innovación cuando enfrenten experiencias similares de montajes en la industria o en la experimentación para la investigación. En este sentido debe crearse un ambiente de aprendizaje donde los estudiantes jueguen un rol más activo; en el que además de la realización de práctica tradicional siguiendo pasos según una guía puedan tener la posibilidad de desarrollar la mayoría de las prácticas como proyectos, planteándosele un problema experimental concreto a resolver (Batista, Mariño, González, 2014).

\section{Metodología}

Para el inicio de este trabajo primeramente se reformaron los programas analíticos de las asignaturas de Física General, Física Aplicada, Calor y Electromagnetismo, Matemática Superior, Cálculo diferencial y Cálculo Integral. Era necesario adecuar estos sílabos a las tendencias actuales acercándolos a lo que generalmente hacen otras universidades y de acuerdo a los requerimientos de calidad según el nuevo régimen académico. Nos era imprescindible después de reformar los de Física comenzar con los de matemática superior, cálculo diferencial y cálculo integral; puesto que era necesario para el cálculo de errores previsto en la mayoría de las prácticas; así como el método de regresión lineal que parte de la optimización de la desviación de 
la recta de ajuste a los datos obtenidos experimentalmente y que expondremos más adelante. El programa analítico de matemática superior no contemplaba el cálculo diferencial e integral que son temas que son tratados desde el bachillerato en la enseñanza de la mayoría de países de la región; lo anterior constituye un problema pues no permite alcanzar el nivel requerido en las asignaturas que se imparten simultáneamente como Física General y Metrología. Posteriormente se definieron las ocho prácticas que se implementarían en cada uno de los temas de las tres asignaturas: Física General, Física Aplicada, Calor y Electromagnetismo. A continuación enumeramos los sistemas de prácticas.

\section{Sistema de prácticas para la asignatura Física general}

P1. Densidad de sólidos, P2. Objetos en equilibrio, P3. Coeficiente de fricción, P4. Coeficiente de viscosidad, P5. Caída libre, P6. Segunda ley de Newton, P7. Trabajo y Energía. P8. Colisiones.

\section{Sistema de prácticas para la asignatura de Física Aplicada a la Ingeniería Automotriz}

P1. Momento de inercia, P2. Oscilaciones mecánicas, P3. Dilatación térmica, P4.Calor específico. P5. Presión hidrostática, P6. Ley de Ohm, P7. Inducción electromagnética, P8. Leyes de la refracción.

\section{Sistema de prácticas para la asignatura Calor y Electromagnetismo.}

P1. Ley de los gases ideales, P2. Calor específico, P3. Primera ley de la termodinámica, P4. Eficiencia de una máquina térmica, P5. Capacitor de placas paralelas, P6. Leyes de Ohm y Kirchhoff, P7. Circuitos RLC en serie y paralelo, P8. Transformadores.

Después de definir las prácticas y los objetivos de cada una de ellas se comenzó a hacer una revisión bibliográfica sobre montajes de prácticas similares para elegir cuáles eran viables y/o reproducibles y cuáles había que diseñar de acuerdo a los recursos disponibles (www.pceiberica.com;www.ventusciencia.com;www.fisicodidactico.com; www.multiciencias.com.ec).

Se buscó un espacio físico, un aula, para adaptarla a las necesidades para la realización y almacenamiento de las prácticas. Se diseñó la estructura que tendría el laboratorio (figura 1).

Se exploró en los laboratorios de electricidad existentes en el campus el equipamiento que no se usaba y que estaban almacenados en los mismos como por ejemplo: fuentes de tensión antiguas, electroimanes, resistencias eléctricas, capacitores, osciloscopios analógicos, generadores de señales analógicos. En el laboratorio de Química se aprovecharon soportes oxidados que no se utilizaban. Se estableció la necesidad de adquirir los componentes que faltaban. En otros casos había que diseñar y mandar a construir otros. 


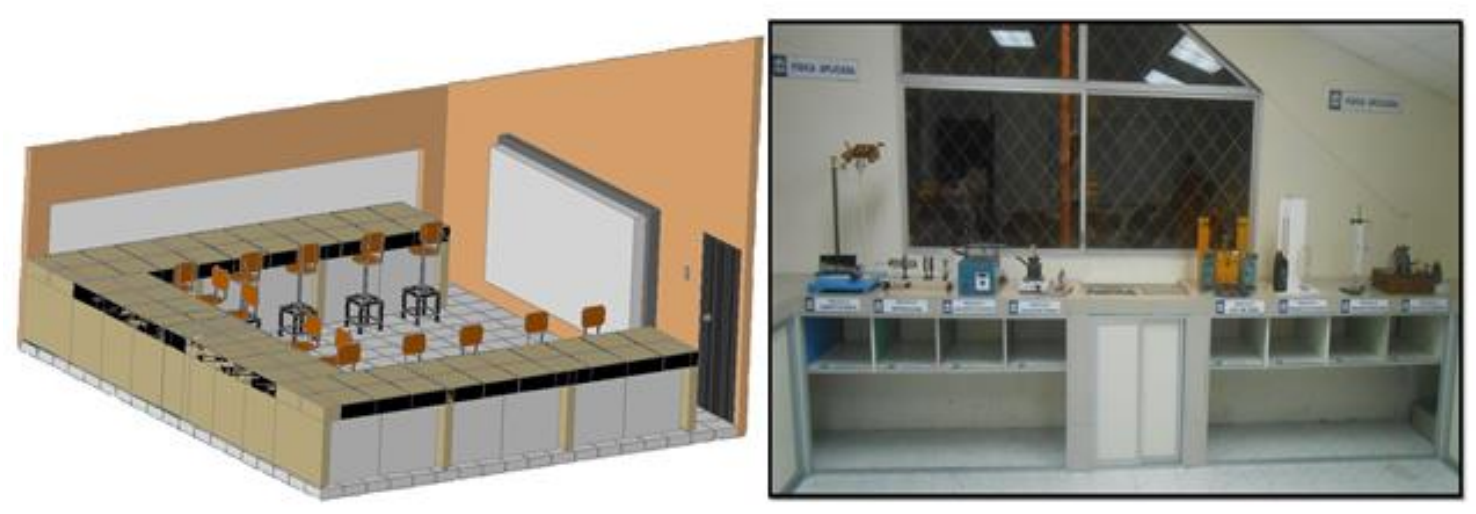

Figura 1. Diseño del espacio para las prácticas.

Se comenzó con el montaje de cada una de las prácticas, se realizaron las pruebas iniciales, se tomaron datos, se aplicaron los métodos de cálculo de errores y/o regresión lineal para comprobar si los resultados estaban acorde con lo que debía obtenerse. En muchos casos hubo que hacer correcciones, rediseños hasta lograr los resultados que debían considerarse como válidos para su implementación definitiva. Se confeccionó una guía para la realización de las prácticas donde constan los detalles y aspectos a tener en cuenta para su correcta manipulación y pasos para cumplir con los objetivos propuestos, no obstante se hace énfasis en que la mayoría de las prácticas se debe enfocar como problemas a resolver donde los estudiantes puedan hacer montajes parciales o totales y propongan cómo resolverán el problema experimental.

Para validar si los resultados de cada práctica estaban en concordancia con los valores previstos en la teoría usamos dos métodos de gran importancia en las ciencias experimentales que son; el método del cálculo del error total y el método de regresión lineal. A continuación exponemos las ideas esenciales de ambos métodos.

El método del error total permite estimar el error tanto en una serie de medidas directas aleatorias así como en las medidas indirectas (Taylor, 2014).

Pasos para determinar el error total de una magnitud $\mathrm{X}$ medida directamente:

1) Se obtiene el error de resolución del instrumento. $\Delta X_{r}$

2) Se mide $\mathrm{N}$ veces la magnitud de interés. $\quad\left(X_{1}, X_{2}, X_{3}, \ldots, X_{N}\right)$

3) Se determina la media aritmética. $\quad \bar{X}=\frac{1}{N} \sum_{i=1}^{N} X_{i}$

4) Se calcula el error estadístico. $\Delta X_{e}=\sqrt{\frac{\sum_{i=1}^{N}\left(\bar{X}-X_{i}\right)^{2}}{N(N-1)}}$ 
5) Se calcula el error total.

$$
\Delta X=\sqrt{\left(\Delta X_{r}\right)^{2}+\left(\Delta X_{e}\right)^{2}}
$$

6) Se expresa la magnitud medida. $\quad X=\bar{X} \pm \Delta X$

Se determina el error relativo.

Cuando se determina indirectamente una $E_{r}=\frac{\Delta X}{\bar{X}} \cdot 100 \%$ magnitud usando una ecuación y sustituyendo en ella medidas directas entonces hay que derivar la ecuación correspondiente para encontrar el error total de la medida indirecta. Por ejemplo sea la $W$ la magnitud que determinaremos experimentalmente dependiente de varias variables esto es:

$$
W=f(x, y, z, \ldots \ldots, n)
$$

Entonces hay que encontrar las derivadas parciales de la función para encontrar el error total de la medida indirecta este método es conocido como propagación de errores.

$$
\Delta W=\sqrt{\left(\frac{\partial W}{\partial x} \cdot \Delta X\right)^{2}+\left(\frac{\partial W}{\partial y} \cdot \Delta Y\right)^{2}+\left(\frac{\partial W}{\partial z} \cdot \Delta Z\right)^{2}+\cdots \cdots+\left(\frac{\partial W}{\partial n} \cdot \Delta N\right)^{2}}
$$

Después de calcular el error total se reporta la medida indirecta: $\quad W=\bar{W} \pm \Delta W$

Se determina además el error relativo:

$$
E_{r}=\frac{\Delta W}{\bar{W}} \cdot 100 \%
$$

El segundo método de regresión lineal es una herramienta de extraordinaria importancia en las ciencias experimentales y en aplicaciones a la ingeniería. Muchas veces dos magnitudes están relacionadas a través de una función matemática conocida y a veces desconocida. A través del análisis de regresión se puede verificar el tipo de dependencia y encontrar de qué forma están relacionadas las variables (Taylor 2014). El caso más sencillo de regresión es la lineal cuando se ajusta una dependencia entres dos variables por medio de una recta. Este tipo de análisis permite estudiar la linealidad entre dos variables. Mediante él se obtiene la recta que tiene una desviación mínima de un conjunto de puntos experimentales. La función objetivo a optimizar es la desviación $S$ y viene dada por la siguiente ecuación:

$$
S=\sum_{i=1}^{N} e_{i}^{2}=\sum_{i=1}^{N}\left(Y_{i}-\left(a \cdot X_{i}+b\right)\right)^{2}
$$


Derivando parcialmente a $\boldsymbol{S}$ con respecto a la pendiente $\boldsymbol{a}$ y al intercepto $\boldsymbol{b}$ se obtienen las ecuaciones para encontrar estos parámetros que son de la recta de mejor ajuste y que generalmente están relacionados con magnitudes físicas de interés que se obtienen experimentalmente.

$$
\frac{\partial S}{\partial a}=0 \cdots a=\frac{N \cdot \sum_{i=1}^{N} X_{i} Y_{i}+\sum_{i=1}^{N} X_{i} \sum_{i=1}^{N} Y_{i}}{N \cdot \sum_{i=1}^{N}\left(X_{i}\right)^{2}-\left(\sum_{i=1}^{N} X_{i}\right)^{2}} \quad \frac{\partial S}{\partial b}=0 \cdots \cdots=\frac{\sum_{i=1}^{N} Y_{i}-a \cdot \sum_{i=1}^{N} X_{i}}{N}
$$

Existen programas de cálculo que dado un conjunto de puntos experimentales (Xi ; Yi) realizan el ajuste obteniéndose estos parámetros. Como por ejemplos: el Microsoft Excell , Grapher y Microlocal Origin. Conjuntamente con este ajuste se obtiene el coeficiente de correlación R que es un indicador de la calidad del ajuste; este coeficiente toma valores comprendidos en el intervalo $(0<R \leq 1]$ para correlaciones positivas y $[-1 \leq R<0)$ para correlaciones negativas y se determina por la ecuación:

$$
R_{X Y}=\frac{\sum_{i=1}^{N} X_{i} Y_{i}-\sum_{i=1}^{N} X_{i} \sum_{i=1}^{N} Y_{i}}{\sqrt{N \cdot \sum_{i=1}^{N}\left(X_{i}\right)^{2}-\left(\sum_{i=1}^{N} X_{i}\right)^{2}} \cdot \sqrt{N \cdot \sum_{i=1}^{N}\left(Y_{i}\right)^{2}-\left(\sum_{i=1}^{N} Y_{i}\right)^{2}}}
$$

Los errores en la determinación de la pendiente y del intercepto vienen dados por las ecuaciones:

$$
\Delta a=\sqrt{\frac{N \cdot \sum_{i=1}^{N}\left(Y_{i}-a X_{i}-b\right)^{2}}{(N-2)\left(N \sum_{i=1}^{N} X_{i}^{2}-\left(\sum_{i=1}^{N} X_{i}\right)^{2}\right)}} \quad \Delta b=\Delta a \cdot \sqrt{\frac{\sum_{i=1}^{N} X_{i}^{2}}{N}}
$$

Estos errores pueden determinarse con el programa Microsoft Excell o mediante un programa de computo sencillo.

\section{Resultados}

En la Figura 2 mostramos el sistema de prácticas para la asignatura de física general (Pazmiño, Valencia, 2014); en la Figura 3 se presenta el sistema de prácticas para la asignatura física aplicada (Bedoya, Jumbo, 2013); En la Figura 4 pueden observase las para la asignatura de Calor y Electromagnetismo (Galván, Ojeda, 2015). En todas ellas se hace una comparación de los costos en tres situaciones: comprando todos los materiales para su montaje, utilizando algunos materiales reciclados y comprando las prácticas ya construidas.

Cada una de las prácticas se validó para su uso mediante medidas experimentales y procesamiento de datos obtenidos obteniéndose las magnitudes de interés para luego compararlas con los valores teóricos y/o resultados esperados. Mostramos a continuación dos ejemplos empleando los métodos de cálculo de errores y regresión lineal expuestos en la introducción de este trabajo. 


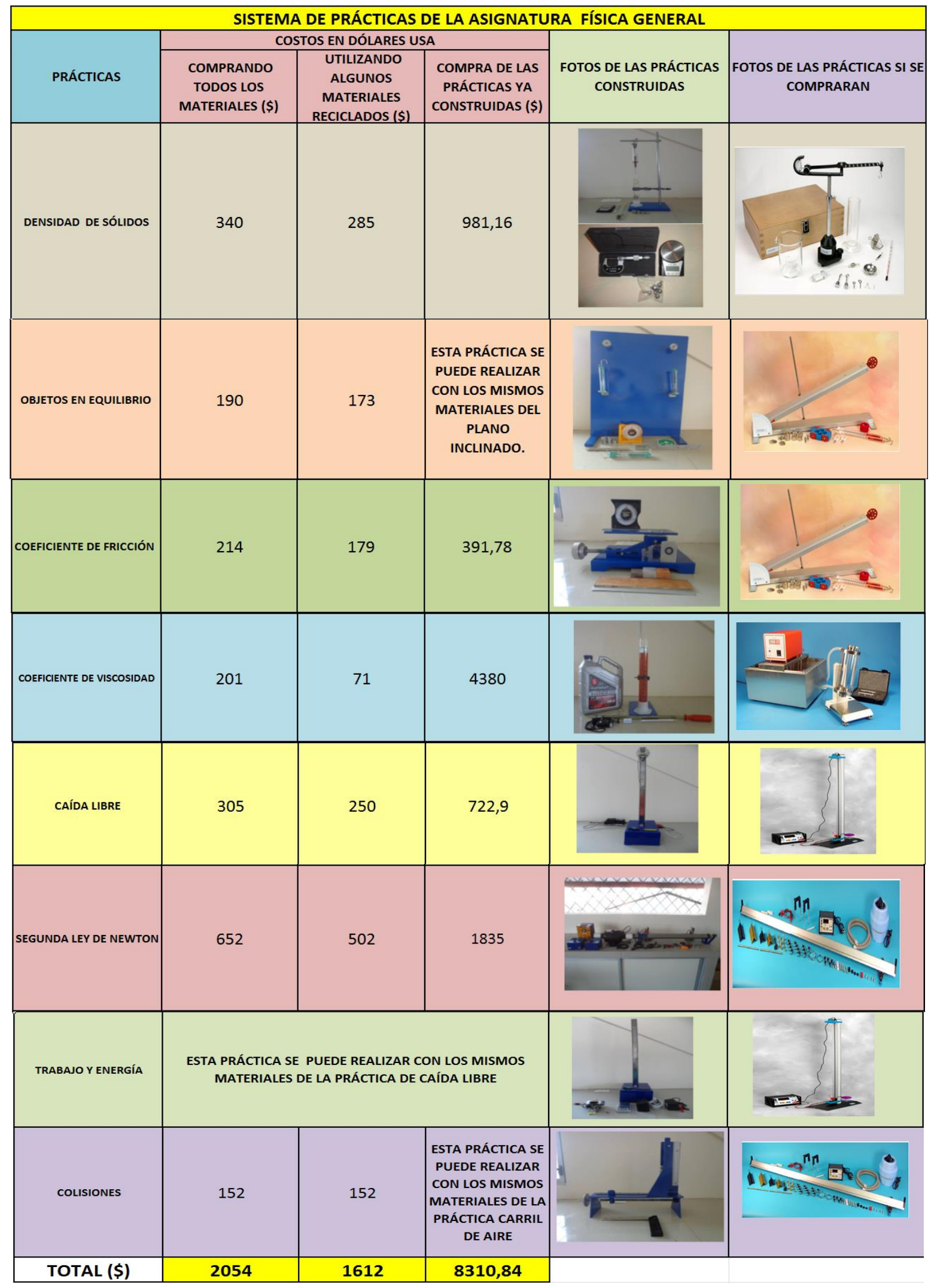

Figura 2. Sistema de prácticas para la asignatura Física General. 


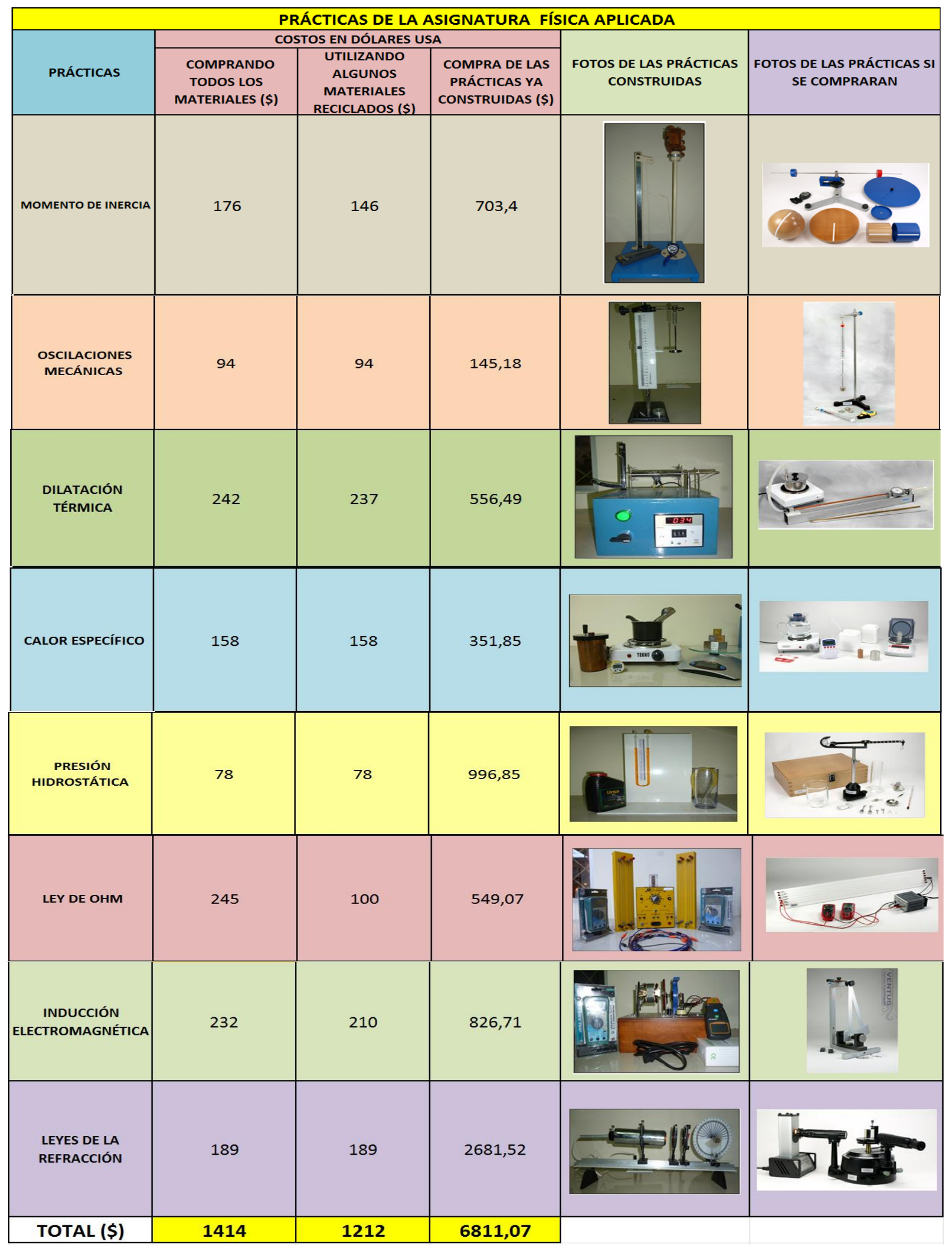

Figura 3. Sistema de prácticas para la asignatura Física Aplicada. 


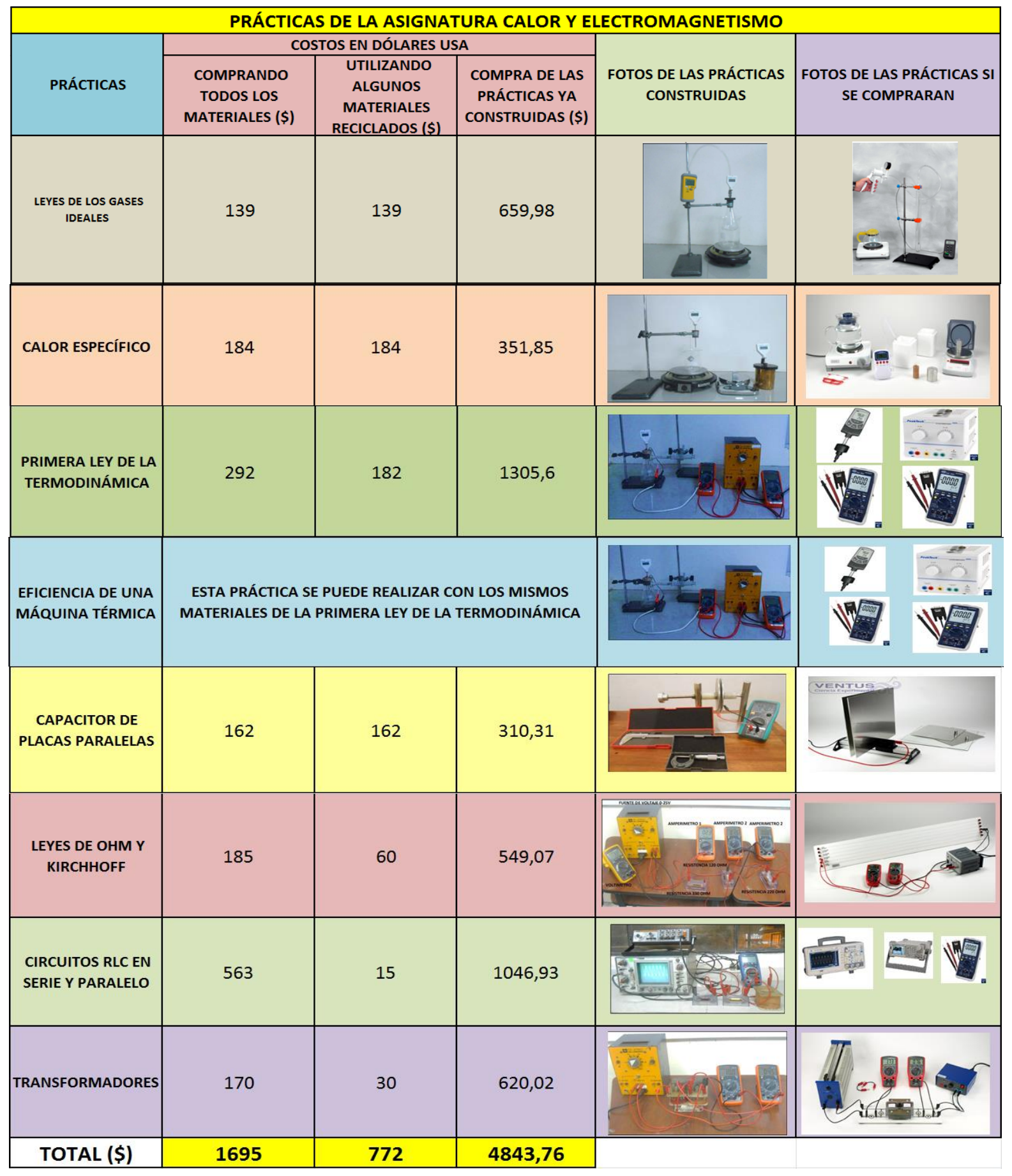

Figura 4. Sistema de prácticas para la asignatura Calor y Electromagnetismo.

Cálculo de errores: El objetivo de la práctica de caída libre de la asignatura de física general es determinar experimentalmente la aceleración de la gravedad y su error total. Mediante la ecuación del movimiento de una partícula bajo la acción de la fuerza de gravedad en caída vertical desde el reposo se obtiene una expresión que relaciona a la aceleración de la gravedad con el tiempo que demora en recorrer cierta distancia:

$$
\bar{g}=\frac{2 \bar{y}}{(\bar{t})^{2}}
$$

Aquí el procedimiento seguido es medir el tiempo (t) que demora una esfera en recorrer una

$$
y=(0,400 \pm 0,001) m
$$


distancia (y) partiendo del reposo. En nuestro caso fijamos la distancia:

En el caso del tiempo procedimos a obtener su valor promedio y el error total siguiendo los pasos descritos con anterioridad para las medidas directas.

1) Obtuvimos el error de resolución del contador digital $\Delta \mathrm{t}_{\mathrm{r}}=0,001 \mathrm{~s}$

2) Medimos en tiempo diez veces; $\mathrm{N}=10$.

$0,286 \mathrm{~s} ; 0,284 \mathrm{~s} ; 0,295 \mathrm{~s} ; 0,277 \mathrm{~s} ; 0,297 \mathrm{~s} ; 0,289 \mathrm{~s} ; 0,282 \mathrm{~s} ; 0,279 \mathrm{~s} ; 0,288 \mathrm{~s} ; 0,287 \mathrm{~s}$

3) Determinamos la media aritmética: $\bar{t}=0,286 \mathrm{~s}$

4) Calculamos el error estadístico: $\Delta t_{\varepsilon}=0,003 \mathrm{~s}$

5) Calculamos el error total: $\Delta t=0,003 \mathrm{~s}$

6) Expresamos el tiempo como: $\quad t=(0,286 \pm 0,003) s$

7) Calculamos el error relativo: $1,048 \%$.

El valor promedio de la aceleración de la gravedad lo calculamos sustituyendo la distancia media y el tiempo medio:

$$
\bar{g}=\frac{2 \bar{y}}{(\bar{t})^{2}}=9,780 \frac{m}{s^{2}}
$$

El error experimental en la determinación de g se calculó aplicando la ecuación de propagación de errores para medidas indirectas, derivando la ecuación de $g$ obtenemos:

$$
\begin{gathered}
\Delta g=\sqrt{\left(\frac{2}{(\bar{t})^{2}} \cdot \Delta y\right)^{2}+\left(-\frac{4 \bar{y}}{(\bar{t})^{3}} \cdot \Delta t\right)^{2}} \\
\Delta y=0,001 m m \quad \bar{t}=0,286 s \quad \bar{y}=0,400 m \quad \Delta t=0,003 s
\end{gathered}
$$

Sustituyendo los valores anteriores: $\Delta g=0,207 \mathrm{~m} / \mathrm{s}^{2}$

Reportamos el valor obtenido como: ${ }^{g=(9,780 \pm 0,207) \mathrm{m} / \mathrm{s}^{2}}$

El error relativo en la determinación de la aceleración de la gravedad es:

$$
\operatorname{Erg}=\frac{\Delta g}{\bar{g}} \cdot 100 \%=\frac{0,207}{9,780} \cdot 100 \%=2,117 \%
$$


Como podemos observar el valor teórico de la aceleración de la gravedad $9,81 \mathrm{~m} / \mathrm{s}^{2}$ pertenece al intervalo encontrado experimentalmente. $[9,573 ; 9,987] \mathrm{m} / \mathrm{s}^{2}$ que se obtiene sumando y restando el error total al valor promedio.

Regresión lineal: El objetivo de la práctica de coeficiente de viscosidad de la asignatura de física general es el de determinar experimentalmente el coeficiente y su error mediante el método de regresión lineal; para ello utilizamos el método de Stokes que consiste en dejar caer una esfera en el líquido y medir la velocidad estacionaria; utilizando la segunda ley de Newton se obtiene una ecuación que relaciona al coeficiente de viscosidad con la velocidad estacionaria y otros parámetros: $\boldsymbol{r}$ radio de la esfera $\boldsymbol{g}$ aceleración de la gravedad, $\boldsymbol{\rho}_{e}$ densidad de la esfera $\boldsymbol{\rho}_{l}$ densidad del líquido, $\boldsymbol{v}_{\mathrm{e}}$ velocidad estacionaria

$$
\begin{gathered}
\mu=\frac{2 \cdot r^{2} \cdot g \cdot\left(\rho_{e}-\rho_{l}\right)}{9 \cdot v_{e}} \\
r=0,000395 m \quad g=9,81 \frac{\mathrm{m}}{\mathrm{s}^{2}} \quad \rho_{e}=7800 \frac{\mathrm{kg}}{\mathrm{m}^{3}} \quad \rho_{l}=870 \frac{\mathrm{kg}}{\mathrm{m}^{3}}
\end{gathered}
$$

La velocidad $\boldsymbol{v}_{\mathrm{e}}$ se determina midiendo el tiempo que tarda la esfera en recorrer una distancia $\mathrm{d}$ en régimen estacionario. $v_{e}=\frac{d}{t}$; sustituyendo en la ecuación del coeficiente de viscosidad:

$$
\mu=\frac{2 \cdot r^{2} \cdot g \cdot\left(\rho_{e}-\rho_{l}\right)}{9 \cdot \frac{d}{t}} \quad, \text { despejando, } \quad d=\frac{2 \cdot r^{2} \cdot g \cdot\left(\rho_{e}-\rho_{l}\right)}{9 \cdot \mu} \cdot t
$$

Si graficamos la distancia en función del tiempo entonces la pendiente de la recta de regresión es $\frac{2 \cdot r^{2} \cdot g \cdot\left(\rho_{e}-\rho_{l}\right)}{9 \cdot \mu}$ de aquí podemos obtener el valor de coeficiente de viscosidad $\mu$.

En la figura mostramos los resultados de las medidas del tiempo que tarda la esfera en recorrer cinco distancias. El ajuste del conjunto de puntos experimentales a una dependencia lineal muestra que el coeficiente de correlación $R=1$ indicando la calidad de las medidas efectuadas y lo apropiado del método para determinar el coeficiente de viscosidad.

Igualando $\frac{2 \cdot r^{2} \cdot g \cdot\left(\rho_{e}-\rho_{l}\right)}{9 \cdot \mu}=0,0089 \frac{m}{s}$ y sustituyendo el resto de parámetros obtenemos que el coeficiente de viscosidad determinado es :

$$
\mu=0,265 \mathrm{~Pa} \cdot \mathrm{s}
$$


El error de la pendiente calculado con la ecuación es prácticamente despreciable del ajuste:

$$
\Delta m=2,4107 \cdot 10^{-8} \mathrm{~m} / \mathrm{s}
$$

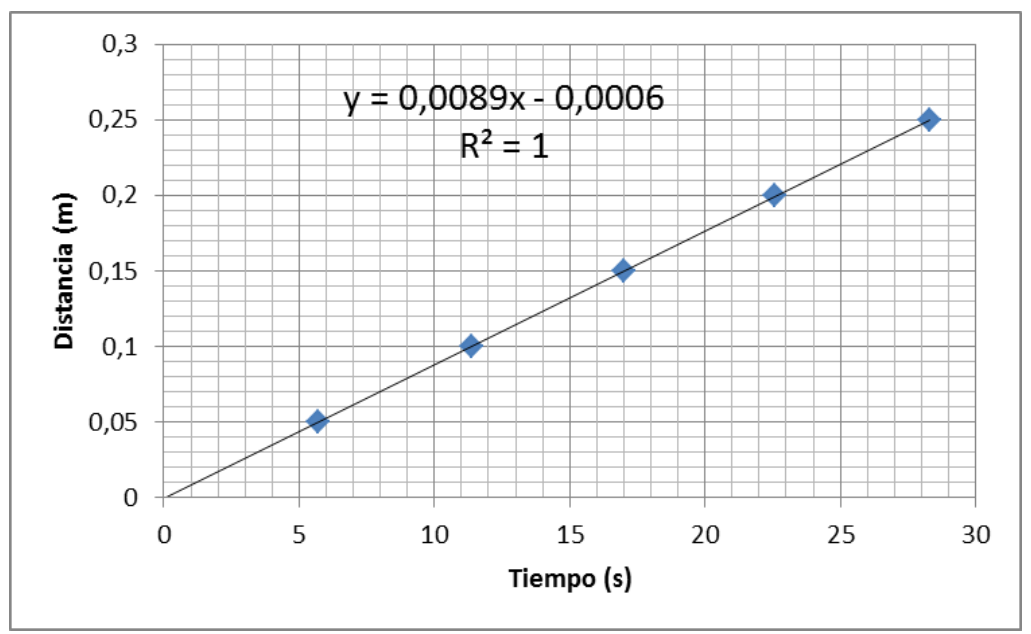

Figura 5. Ajuste por el método de regresión lineal.

El coeficiente de viscosidad fue determinado a partir de la pendiente de la recta por lo tanto estará influenciado por el error de esta y de los demás parámetros como el error de radio, la aceleración de la gravedad y de la medición de las densidades. Aplicando la ecuación de propagación de errores obtenemos:

$$
\Delta \mu=\sqrt{\left(\frac{4 r g \cdot \delta \rho}{9 m} \cdot \Delta r\right)^{2}+\left(\frac{2 r^{2} \cdot \delta \rho}{9 m} \cdot \Delta g\right)^{2}+\left(\frac{2 r^{2} g}{9 m} \cdot \Delta \rho\right)^{2}+\left(-\frac{2 r^{2} g \cdot \delta \rho}{9 m^{2}} \cdot \Delta m\right)^{2}}
$$

En la ecuación anterior:

$\mathrm{r}=0,000395 \mathrm{~m}$, es el radio de la esfera; $\Delta \mathrm{r}=0,00001 \mathrm{~m}$, es su error.

$\mathrm{g}=9,81 \frac{\mathrm{m}}{\mathrm{s}^{2}}$, es la aceleración de la gravedad; $\Delta \mathrm{g}=0,01 \frac{\mathrm{m}}{\mathrm{s}^{2}}$, es su error.

$\delta \rho=\rho_{\mathrm{e}}-\rho_{\mathrm{l}}=6930 \frac{\mathrm{kg}}{\mathrm{m}^{3}}$, es la diferencia de densidades $; \rho=10 \frac{\mathrm{kg}}{\mathrm{m}^{3}}$, es su error.

$\mathrm{m}=0.089 \frac{\mathrm{m}}{\mathrm{g}}$, es la pendiente (velocidad estacionaria); $\Delta \mathrm{m}=2,42 \cdot 10^{-8} \frac{\mathrm{m}}{\mathrm{g}}$, es su error.

Al sustituir todos los términos en la ecuación anterior obtenemos un error para el coeficiente de viscosidad de:

$$
\Delta \mu=0,014 \mathrm{~Pa} \cdot \mathrm{s}
$$


El error relativo en la determinación del coeficiente de viscosidad:

$$
E r \mu=\frac{\Delta \mu}{\mu} \cdot 100 \%=\frac{0,014}{0,265} \cdot 100 \%=5,283 \%
$$

En la Tabla 1 hacemos una comparación entre el resultado esperado en cada práctica con el resultado obtenido nos referimos a tres aspectos si es válida, el error que se obtiene, y la linealidad esperada según el objetivo de cada práctica.

Tabla 1. Cuantificación de errores

\begin{tabular}{|c|c|c|c|}
\hline Prácticas & Resultado esperado & Resultado Obtenido & $\begin{array}{c}\text { Validez } \\
\text { Error } \\
\text { Linealidad }\end{array}$ \\
\hline Densidad de sólidos & Densidad acero, $7800 \mathrm{~kg} / \mathrm{m}^{3}$ & $7780 \mathrm{~kg} / \mathrm{m}^{3}$ & $0,26 \%$ \\
\hline Objetos en equilibrio & $\mathrm{T}_{1}=1,44 \mathrm{~N} ; \mathrm{T}_{2}=0,96 \mathrm{~N}$ & $\mathrm{~T}_{1}=1,40 \mathrm{~N} ; \mathrm{T}_{2}=1,00 \mathrm{~N}$ & 2,$78 ; 4,17 \%$ \\
\hline Coeficiente de fricción & {$[0,15 ; 0,60]$} & 0,24 & Válido \\
\hline Coeficiente de viscosidad & 0,260 Pa.s & 0,265 Pa.s & $1,93 \%$ \\
\hline Caída libre & $9,81 \mathrm{~m} / \mathrm{s}^{2}$ & $9,78 \mathrm{~m} / \mathrm{s}^{2}$ & $0,31 \%$ \\
\hline Segunda ley de Newton & linealidad F vs aceleración & $\mathrm{F}=0,1359 \mathrm{a}+0,0195(\mathrm{~N})$ & $r=0,99$ \\
\hline Trabajo y Energía & $E_{c}=E_{p}=0,084167 \mathrm{~J}$ & $E_{c}=0,08155 \mathrm{~J}$ & $3,11 \%$ \\
\hline Colisiones & $P_{f}=P_{i}=0,025 \mathrm{kgm} / \mathrm{s}$ & $P_{f}=0,023 \mathrm{kgm} / \mathrm{s}$ & $8,00 \%$ \\
\hline Momento de inercia & $I_{x}>I_{y}>I_{z}$ & $\begin{array}{l}0,2791>0,1989>0,0828 \\
\left(\mathrm{~kg} \cdot \mathrm{m}^{2}\right)\end{array}$ & Válido \\
\hline Oscilaciones mecánicas & $\begin{array}{l}\text { Linealidad; } \\
\beta=0,0200 \mathrm{~s}^{-1}\end{array}$ & $\begin{aligned} \operatorname{Ln}\left(A_{t} / A_{0}\right) & =-0,0203 \cdot t-0,1745 \\
\beta & =0,0203 \mathrm{~s}^{-1}\end{aligned}$ & $\begin{array}{c}r=0,95 \\
1 \%\end{array}$ \\
\hline Dilatación térmica & Aluminio $2,40 \times 10^{-5}{ }^{\circ} \mathrm{C}^{-1}$ & $2,43 \times 10^{-5}{ }^{\circ} \mathrm{C}^{-1}$ & $1,25 \%$ \\
\hline Calor específico & Aluminio $\mathrm{c}=900 \mathrm{~J} / \mathrm{kg} . \mathrm{K}$ & $881 \mathrm{~J} / \mathrm{kg} . \mathrm{K}$ & $3 \%$ \\
\hline Presión hidrostática & $\begin{array}{c}\text { Linealidad } \\
\rho_{\text {aceite }}=870 \mathrm{~kg} / \mathrm{m}^{3}\end{array}$ & $\begin{array}{c}\mathrm{h}_{2}=0,927 \mathrm{~h}_{1}-0,0004(\mathrm{~m}) \\
\rho_{\text {aceite }}=917 \mathrm{~kg} / \mathrm{m}^{3}\end{array}$ & $\begin{array}{c}r=0,99 \\
5 \%\end{array}$ \\
\hline Ley de Ohm & $\begin{array}{c}\text { Linealidad, } V=R . I \\
R=1000 \Omega\end{array}$ & $\begin{array}{c}\mathrm{V}=1014,4 \cdot \mathrm{I}-0.0064(\mathrm{~V}) \\
\mathrm{R}=1014,4 \Omega\end{array}$ & $\begin{array}{c}r=1 \\
1,4 \%\end{array}$ \\
\hline Inducción electromagnética & $\begin{array}{c}\text { Linealidad, } B=30,00 \mathrm{~T} \\
B=30,8 \mathrm{~T}\end{array}$ & $\begin{array}{c}\varepsilon_{i}=0,002 \cdot \omega+0,0026(V) \\
B=31,8 \mathrm{~T}\end{array}$ & $\begin{array}{l}r=1 \\
6 \%\end{array}$ \\
\hline Leyes de la refracción & Índice refracción, $n=1,50$ & $\mathrm{n}=1,57$ & $4,67 \%$ \\
\hline Leyes de los gases ideales & Linealidad, $P=f(T)$ & $\mathrm{P}=43,484 \mathrm{~T}-12615(\mathrm{~Pa})$ & $r=0,99$ \\
\hline Calor específico & Aluminio $\mathrm{c}=900 \mathrm{~J} / \mathrm{kg} . \mathrm{K}$ & $849 \mathrm{~J} / \mathrm{kg} . \mathrm{K}$ & $6 \%$ \\
\hline Primera ley termodinámica & $\dot{Q}=\dot{W}+\Delta \dot{U}$ & $105,95=80,63+20,34(\mathrm{~W})$ & $4,70 \%$ \\
\hline Eficiencia máquina térmica & Eficiencia $\Pi<100 \%$ & $\eta=10 \%$ & válido \\
\hline $\begin{array}{l}\text { Capacitor de placas } \\
\text { paralelas }\end{array}$ & $\begin{array}{c}\text { Linealidad } \\
\text { Permitividad relativa, } \varepsilon_{r}=4,5\end{array}$ & $\begin{array}{l}\mathrm{C}=40,729 \cdot \mathrm{A} / \mathrm{d}-0,346(\mathrm{nF}) \\
\text { Permitividad relativa, } \varepsilon_{\mathrm{r}}=4,1\end{array}$ & $\begin{array}{c}r=0,97 \\
10 \%\end{array}$ \\
\hline Leyes de Ohm y Kirchhoff & $\begin{array}{c}\text { Linealidad } \\
\mathrm{R}=137 \Omega \\
\mathrm{I}=\mathrm{I}_{1}+\mathrm{I}_{2} \\
\mathrm{~V}=\mathrm{V}_{1}+\mathrm{V}_{2}\end{array}$ & $\begin{array}{c}\mathrm{V}=135,94 \mathrm{I}+0,0223(\mathrm{~V}) \\
\mathrm{R}=135,94 \Omega \\
2,3=1,5+0,8(\mathrm{~A}) \\
2,06=1,63+0,43(\mathrm{~V})\end{array}$ & $\begin{array}{c}r=0,99 \\
0,78 \% \\
0 \% \\
0 \%\end{array}$ \\
\hline $\begin{array}{llll}\begin{array}{l}\text { Circuito } \\
\text { paralelo }\end{array} & & & \\
\end{array}$ & $f$ de resonancia, $f=870 \mathrm{~Hz}$ & $\mathrm{f}=900 \mathrm{~Hz}$ & $3,45 \%$ \\
\hline Transformadores & $\mathrm{K}_{\text {elevador }}=2 ; \mathrm{k}_{\text {reductor }}=0,5$ & $\mathrm{~K}_{\text {elevador }}=2,14 ; \mathrm{k}_{\text {reductor }}=0,53$ & $7 \% ; 6 \%$ \\
\hline
\end{tabular}




\section{Discusión}

Analizando la Figura 2 vemos como en las prácticas de física general se obtiene un ahorro de \$6698,84; en la Figura 3 para el caso de física aplicada el ahorro es de \$ 5599 y en la Figura 3 correspondientes a las de calor y electromagnetismo es de unos $\$ 4071,73$. El ahorro total en el montaje de las 24 prácticas de los tres sistemas es de unos \$16369,57 comparado con el hecho de si se hubiesen comprado cada una de las prácticas ya montadas.

En la validación de las prácticas para el uso docente en doce de ellas se obtienen errores por debajo del $3 \%$, lo cual es un resultado satisfactorio, estas prácticas sirven para estimar parámetros desconocidos con buena precisión. En otras ocho prácticas se obtiene la linealidad entre parámetros de leyes físicas lo cual sirve para el estudio y comprensión de fenómenos. En cuatro prácticas se obtienen resultados válidos o sea aceptables pues los valores obtenidos experimentalmente se encuentran dentro del rango previsto.

\section{Conclusiones y Recomendaciones}

En este trabajo no hay aportes científicos porque el tema es bastante conocido hay empresas que comercializan y todo lo referente al diseño está bien concebido, pero a nivel de prácticas para la docencia no es necesario un grado de precisión elevado como el que si requieren los laboratorios de investigaciones científicas, entonces los aportes de este trabajo son en el orden práctico para la docencia y en el aspecto económico.

Con la realización de este trabajo quedó implementado un laboratorio de Física con los tres sistemas de prácticas previstos que sin duda contribuyen al desarrollo de habilidades experimentales en los estudiantes de la misma manera que si se hubiesen comprado cada uno de los montajes.

Son significativos, el ahorro económico para la institución al haberse concluido el montaje del laboratorio y también la posibilidad de que los estudiantes tengan una cobertura para la realización de su tesis dentro de la universidad.

Este trabajo puede servir de referencia para que profesores y estudiantes de la universidad y otras instituciones exploren y aprovechen el potencial existente en ellas para implementar prácticas de laboratorio en asignaturas de ciencias básicas e incluso de especialización en diferentes carreras de ingeniería.

Es imprescindible el apoyo de las autoridades académicas para que puedan materializarse las iniciativas que contribuyan a este tipo de trabajos cuyo único fin es el de elevar el nivel y la calidad de la enseñanza mediante el desarrollo de habilidades experimentales. 


\section{Bibliografía}

Agudelo, J.D., García, D.(2010). Aprendizaje significativo a partir de prácticas de laboratorio de precisión. Latin - American Journal of Physics Education, 149-152.

Flores, J., Caballero Sahelices, M.C., Moreria, M.A.(2009). El laboratorio en la enseñanza de las ciencias: una visión integral en este complejo ambiente de aprendizaje. Revista Investigación, 75-111.

Gil, S.(2015). Experimentos de física usando las tic y elementos de bajo costo. Marcombo, S.A.

Batista, M.A; Mariño, P.A; González S.I (2014) . Los laboratorios-proyectos como estrategia didáctica a través del desarrollo de competencias investigativas. Revista Cubana de Física,VOL31, E72,37-38.

Taylor, J.R (2014). Introducción al análisis de Errores: el estudio de las incertidumbres en las mediciones físicas. Editorial Reverté, 1aㅡ Edición.

Pazmiño, L; Valencia, D. (2014). Implementación de un laboratorio y de un sistema de prácticas para la asignatura de Física General en La Universidad tecnológica Equinoccial Extensión Santo Domingo. Tesis para optar por el título de Ingeniero Electromecánico.

Bedoya, C; Jumbo, J. (2013). Diseño e Implementación de un sistema de prácticas de laboratorio para la asignatura de Física Aplicada de la carrera de Ingeniería Automotriz en la Universidad Tecnológica Equinoccial Santo Domingo. Tesis para optar por el título de Ingeniero Electromecánico.

Galván, M; Ojeda, O. (2015). Diseño e implementación de un sistema de prácticas para la asignatura Calor y Electromagnetismo de la carrera de Ingeniería Electromecánica en la Universidad Tecnológica Equinoccial Santo Domingo. Tesis para optar por el título de Ingeniero Electromecánico.

\section{Agradecimientos:}

A: Joaquin Morales LLumiquinga, prorector de la sede Santo Domingo de la Universidad Tecnológica Equinoccial; Marcelo Estrella Guayasamín, Nilo Olegario Ortega Soliz, coordinadores de las carreras de Ingeniería Automotriz y Electromecánica, quienes aceptaron la iniciativa y propiciaron las condiciones para que pudiera implementarse el laboratorio. A los estudiantes y hoy ingenieros: Diego Fabián Valencia Alvear, Luis Geovanny Pazmiño Bravo, Chistopher Alexander Bedoya Torres, José Fabián Jumbo Román, Mayra Mariuxi Galván Paredes y Oscar Oswaldo Ojeda Carrera; quienes con su trabajo y aporte pusieron a punto el laboratorio de Física en la sede Santo Domingo de la Universidad Tecnológica Equinoccial. 